\title{
Principais efeitos colaterais das drogas antiobsessivas e seu manejo
}

\author{
A ristides $V$ Cordioli \\ Departamento de Psiquiatria e Medicina Legal da Universidade Federal do Rio Grande do Sul. Porto Alegre, RS, Brasil
}

\begin{abstract}
Selective serotonin reuptake inhibitors (SSRIs), clomipramine, and behavior therapy are the first line treatments for obsessive-compulsive (OCD) symptoms. One of the main reasons for treatment non-compliance and early discontinuation of medications by patients or premature change in prescriptions by clinicians are their side effects. Nausea, headache, insomnia, restlessness, sexual dysfunction, and tremors are common with SSRIs; hypotension, dry mouth, constipation, urinary retention, blurred vision, weight gain, sedation and cardiocirculatory problems, with clomipramine. The strategies and guidelines for these drugs' side effects management are here reviewed, based mainly in case reports and clinical experience. The clinician should be aware of the importance of addressing this issue, educating patients about the most frequent adverse reactions, and how to deal with them in order to prevent unwanted medication discontinuation, and enhance compliance with the treatment.
\end{abstract}

Keywords Obsessive-compulsive disorder. Treatment. Selective serotonin reuptake inhibitors. Clomipramine. Adverse effects. Management.

\section{Introdução}

Os efeitos colaterais das drogas antiobsessivas são a principal razão da não adesão ao tratamento e de mudanças precipitadas de medicação. ${ }^{1}$ Algumas medidas podem evitar tais incidentes: informar ao paciente sobre os efeitos mais freqüentes e sobre o fato de que eles podem diminuir ou desaparecer depois das primeiras semanas de uso; aumentar ou diminuir gradualmente as doses; tomar medicamentos que causam náuseas ou vômitos junto às refeições, pela manhã, os que causam insônia, e, à noite, os que provocam sonolência; dividir a dose diária em duas ou mais tomadas ou trocar de medicamento. Deve-se sempre levar em conta ao prescrever: idade, sensibilidade individual, perfil de sintomas que o paciente apresenta (aumento de peso, insônia, ansiedade etc.), doenças físicas existentes, uso concomitante de outras drogas, especialmente as que alteram o metabolismo hepático, pois tais fatores influem na ocorrência de reações adversas. Serão apresentados os efeitos colaterais mais comuns das drogas antiobsessivas e sugeridas medidas para o seu manejo.

\section{Efeitos cardiocirculatórios Hipotensãa postural}

É comum com o uso da clomipramina e rara com os inibidores seletivos de recaptação da serotonina (ISRS): queda da pressão arterial por bloqueio $\alpha 1$-adrenérgico, provocada por mudança súbita de posição, acompanhada de tonturas. Ocorre mais em pacientes idosos ou debilitados, em dieta hipossódica, desidratados ou em restrição líquida, utilizando antihipertensivos ou diuréticos, com hipotiroidismo ou hipofunção adrenal. Tende a ser mais grave quando há insuficiência cardíaca, bloqueio de ramo ou arritmias. Medidas: a) evitar levantar-se da cama, da cadeira ou abaixar-se bruscamente;

b) evitar banhos quentes prolongados;

c) evitar refeições pesadas e álcool;

d) usar meias elásticas de pressão média;

e) ingerir cloreto de sódio ( $3 \mathrm{~g} / \mathrm{dia}$ a $4 \mathrm{~g} /$ dia) ou fluidrocortisona (0,1-0,5 mg uma a três vezes ao dia), monitorando a tensão arterial e o edema.

f) substituir clomipramina por ISRS.

\section{Alterações no ritmo cardíaco}

A clomipramina pode provocar taquicardia e extra-sístoles em função de seus efeitos anticolinérgicos. Os ISRS, em princípio, não afetam a condução cardíaca, mesmo em pacientes com problemas cardíacos preexistentes. ${ }^{2}$ Recomenda-se:

a) evitar o uso de clomipramina em pacientes com problemas cardíacos, crianças e idosos;

b) evitar a fluoxetina em pacientes com bloqueio de ramo ou doença coronariana que estejam tomando propranolol ou metoprolol: pode ocorrer bradicardia severa. ${ }^{3,4}$

\section{Efeitos anticolinérgicos \\ Boca seca}

Comum com a clomipramina pela redução da produção de saliva e rara com os ISRS (ocorrendo mais com a paroxetina). Medidas:

a) mascar chicletes ou chupar balas dietéticas;

b) bochechos com pilocarpina a $1 \%$, uma hora antes de palestras, aulas, conversação intensa etc.;

c) $10 \mathrm{mg}$ a $30 \mathrm{mg}$ betanecol, três vezes ao dia; 
d) manter boa higiene bucal, visitas regulares ao dentista e evitar açúcar (cáries);

e) trocar de medicamento.

\section{Constipação intestinal}

Muito comum com a clomipramina pelos seus efeitos anticolinérgicos e rara com os ISRS. Medidas:

a) dieta rica em fibras ou substâncias que retenham líquidos no intestino, como mucilóide de Psyllium, 1 a 2 envelopes/dia;

b) aumentar a ingestão de líquidos;

c) praticar exercícios físicos regularmente;

d) caso essas medidas não sejam suficientes: laxativos de contato, como picossulfato de sódio, 8 gotas/dia a 15 gotas/ dia, ou laxantes naturais não-irritantes do intestino; ${ }^{5}$

e) substituir clomipramina por um ISRS.

\section{Retenção urinária}

Dificuldade de iniciar a micção como conseqüência de ações anticolinérgicas sobre o esfíncter vesical, podendo provocar retenção urinária, alargamento da bexiga e infecções. Medidas:

a) betanecol, $10 \mathrm{mg}$ VO a $30 \mathrm{mg}$ VO, três vezes ao dia;

b) evitar o uso de clomipramina e paroxetina em pacientes com história de retenção urinária (p. ex.: hiperplasia prostática).

\section{Visão borrada}

Conseqüência da dificuldade de acomodação visual por efeito anticolinérgico. Comum com a clomipramina, rara com os ISRS. Pode-se:

a) reduzir a dose ou tentar outra droga que não produza esse efeito;

b) aplicar colírio de pilocarpina a 1\% - algumas gotas duas a três vezes ao dia;

c) usar betanecol, $10 \mathrm{mg} \mathrm{VO}$ a $30 \mathrm{mg}$ VO, três vezes/dia.

\section{Disfunções sexuais}

Diminuição da libido e da excitação, retardo na ejaculação ou anorgasmia ocorrem em $58 \%$ a $73 \%$ dos pacientes que utilizam drogas antiobsessivas. Dificilmente desaparecem espontaneamente, comprometendo a qualidade de vida e a adesão ao tratamento. ${ }^{6,7}$ Ocorrem provavelmente pelo bloqueio de receptores $\alpha$-adrenérgicos e estimulação crônica de receptores 5HT2. Questionar sempre o paciente quanto à presença desses sintomas, geralmente omitidos. Caso sejam leves ou intermitentes, pode-se aguardar para ver se desaparecem ou diminuem espontaneamente. Pode-se também diminuir a dose, trocar de medicamento ou usar um antídoto. Com exceção do sildenafil, que pode ser tomado uma hora antes da relação sexual, recomendase que os demais antídotos sejam utilizados de forma contínua, suspendendo-se periodicamente para observar se o problema desapareceu. $^{8}$

\section{Antídotos}

Diminuição da libido, excitação, retardo no orgasmo ou anorgasmia:

a) sildenafil: $50 \mathrm{mg}$ a $100 \mathrm{mg} 1$ hora antes da relação sexual. ${ }^{9}$ Parece ser efetivo mesmo em mulheres; b) ioimbina - um agonista $\alpha$-adrenérgico: $5 \mathrm{mg}$ a $10 \mathrm{mg} 1$ a 2 horas antes da relação sexual ou $5 \mathrm{mg}$ três vezes ao dia;

c) bupropriona: $75 \mathrm{mg}$ a $150 \mathrm{mg} 1$ a 2 horas antes da relação ou $75 \mathrm{mg}$ três vezes ao dia; ${ }^{7}$

d) extrato de gingko biloba: $180-240 \mathrm{mg} / \mathrm{dia}$, em duas doses diárias.

\section{Retardo no orgasmo ou anorgasmia}

a) nefazodona: $50 \mathrm{mg} /$ dia a $150 \mathrm{mg} /$ dia; mirtazapina: $15 \mathrm{mg} /$ dia a $30 \mathrm{mg} / \mathrm{dia}$ ou uma hora antes do coito. Usar com cuidado a associação de nefazodona com fluoxetina.

b) ciproeptadina: $4 \mathrm{mg} / \mathrm{dia}$ a $12 \mathrm{mg} / \mathrm{dia}^{1} 1$ a 2 horas antes do coito;

c) amantadina - um agonista dopaminérgico: $100 \mathrm{mg}$ a $200 \mathrm{mg}$ duas vezes ao dia ou $100 \mathrm{mg}$ 5-6 horas antes do coito.

\section{Retardo na ejaculação}

Comum com a clomipramina, raro com os ISRS. Aparentemente não ocorre com a fluvoxamina. ${ }^{10}$ Inicia-se ao redor de uma semana depois do início da droga, diminuindo muito pouco com o tempo. Deve-se a bloqueio de receptores $\alpha 1$-adrenérgicos e 5HT1a. Pode-se:

a) mudar de droga;

b) associar 7,5 mg a 15,0 mg de neostigmina, 30 minutos antes da relação;

c) bupropiona (75 mg a $100 \mathrm{mg}$ ) ou nefazodona (100 mg a 200 mg) 1 a 2 horas antes da relação.

\section{Efeitos gastrointestinais}

\section{Náuseas, vômitos, dispepsia}

Comuns com os ISRS, raros com a clomipramina. Em geral são leves e desaparecem após algumas semanas. Medidas:

a) iniciar com doses menores ou reduzi-las, ingerindo-as durante as refeições ou à noite;

b) náuseas severas e vômitos: $5 \mathrm{mg}$ a $10 \mathrm{mg}$ duas vezes ao dia de cisaprida, retirando gradualmente depois de algumas semanas.

\section{Ganho de peso}

Decorre do aumento do apetite, da fissura por doces e das mudanças na regulação das reservas de gordura do organismo, em função da modulação do sistema hipotalâmico conseqüente ao bloqueio de receptores H1 e 5HT2C. É comum com a clomipramina. Os ISRS em geral provocam uma perda de peso em curto prazo, podendo haver ganho em longo prazo especialmente com a paroxetina. ${ }^{11,12}$ Em princípio não ocorreria com o uso crônico da fluoxetina, ${ }^{13}$ o que é controverso. Medidas:

a) dieta hipocalórica e exercícios físicos;

b) substituir a clomipramina por fluoxetina ou sertralina.

\section{Sistema nervoso central Ansiedade, inquietude}

Tanto a clomipramina quanto os ISRS podem provocar, no início do tratamento, inquietude, tensão, ansiedade e dificuldade para relaxar. Portanto, recomenda-se:

a) iniciar com doses baixas ou reduzi-las ( $5 \mathrm{mg}$ de fluoxetina ou 10 mg de clomipramina) e aumentar lentamente até atingir os níveis recomendados; 
b) caso persistam os sintomas, associar clonazepam ( $1 \mathrm{mg} / \mathrm{dia}$ a $4 \mathrm{mg} /$ dia $)$ ou alprazolam $(0,5-4 \mathrm{mg} /$ dia $)$ e retirar gradualmente à medida que forem desaparecendo;

c) propranolol (20-80 mg/dia).

\section{Insônia}

A estimulação de receptores 5HT2 e alterações na arquitetura do sono parecem ser as causas da insônia causada pelos ISRS. ${ }^{14}$

a) administrar o medicamento de preferência pela manhã;

b) se não houver contra-indicações, preferir clomipramina ou paroxetina;

c) adicionar pequenas doses diárias de trazodona (25-100 mg) ou mirtazapina (15-30 mg);

d) associar benzodiazepínicos por pequenos períodos de tempo ou zolpidem $(10 \mathrm{mg}) .{ }^{15}$

\section{Sonolência}

Sonolência diurna é mais comum com a clomipramina e a paroxetina. Podem ocorrer bocejos com ausência de sono que, em geral, desaparecem espontaneamente. Recomenda-se:

a) iniciar com doses baixas à noite, pois normalmente desenvolve-se tolerância a esse efeito depois de 2 a 4 semanas;

b) preferir a fluoxetina e a sertralina;

c) aumentar o uso de café ou chá;

d) evitar o uso associado de outros depressores do SNC, como álcool e benzodiazepínicos;

e) associar bupropriona ( $75 \mathrm{mg} / \mathrm{dia}$ a $150 \mathrm{mg} / \mathrm{dia})$ ou dextroanfetamina $5 \mathrm{mg} / \mathrm{dia}$ a $30 \mathrm{mg} / \mathrm{dia}$.

\section{Mioclonias}

São abalos musculares involuntários que ocorrem geralmente à noite, podendo interferir no sono. Clonazepan, 0,5 $\mathrm{mg}$ a $2 \mathrm{mg}$ antes de deitar, ou outro benzodiazepínico podem abolir o sintoma.

\section{Tremores}

Podem ocorrer no uso da clomipramina e dos ISRS:

a) suspender o uso de café;

b) diazepam, $5 \mathrm{mg}$ a $10 \mathrm{mg}, 2$ vezes/dia;

Em pacientes livres de cardiopatias:

c) propranolol, $10 \mathrm{mg}$ a $20 \mathrm{mg}, 3$ vezes $/$ dia - até $80 \mathrm{mg} / \mathrm{dia}$ a 120 $\mathrm{mg} / \mathrm{dia}$;

d) metoprolol 100-200 mg/dia em doses divididas ou nadolol, quando se prefere a eliminação renal à hepática.

\section{Síndrome de descontinuação}

Pode ocorrer em função da redução abrupta, do esquecimento de doses ou da descontinuação da clomipramina e dos ISRS. Os sintomas mais comuns são ansiedade, agitação, insônia, tonturas, vertigens, fadiga, náuseas, dores musculares, coriza, mal-estar, perturbações sensoriais e depressão. Iniciam 12 a 48 horas após a última dose, durando em geral até duas semanas. ${ }^{16-18}$ Ocorre com mais freqüência na descontinuação da paroxetina, seguida do citalopram, da sertralina e da fluvoxamina. Praticamente não ocorre com a fluoxetina. ${ }^{19,20}$ É recomendável:

a) alertar o paciente para o risco da síndrome se por acaso esquecer de tomar o medicamento ou interrompê-lo abruptamente;

b) reintroduzir uma única dose do fármaco para eliminar os sintomas e proceder à retirada gradual;

c) em pacientes pouco aderentes, preferir fármacos de meiavida longa (fluoxetina).

\section{Conclusões}

A adesão do paciente ao tratamento é essencial para o seu sucesso. As reações adversas são a principal razão de abandono ou mudança precoce de medicamento. Dar a devida atenção a essa questão, orientando o paciente e pondo-se à disposição para esclarecer dúvidas a qualquer momento são atitudes essenciais, especialmente no início da terapia, podendo evitar tais insucessos.

\section{Referências}

1. Koran LM. Obsessive-compulsive disorders in adults: a comprehensive clinical guide. Cambridge: Cambridge University Press; 1999.

2. Glassman AH. Cardiovascular effects of antidepressant drugs: updated. J Clin Psychiatry 1998;59(supll 15):13-8.

3. Walley T, Pirmohamed M, Proudlove C, Maxwell D. Interaction of metoprolol and fluoxetine [letter]. Lancet 1993;341:967-8.

4. Drake WM, Gordon GD. Heart block in a patient on propranolol and fluoxetine. Lancet 1994;343:425-6.

5. Souza NO, Cordioli AV, Shansis EH, Pádua AC, Baroni GV. Efeitos colaterais e seu manejo. In: Cordioli AV, ed. Psicofármacos: consulta rápida. Porto Alegre: Artmed; 2000.

6. Montejo-Gonzales AI, Lhorca G, Rico-Villademoros F. Incidence of sexual dysfunction associated with antidepressants agents: a prospective study of 1022 outpatients. J Clin Psychiatry 2001;62(supll 3):10-21.
7. Rothschild AJ. Sexual side effects of antidepressants. J Clin Psychiatry 2000;61(supll 11):28-36.

8. Zajecka J. Clinical issues in long-term treatment with antidepressants. J Clin Psychiatry 2000;61(supll 2):20-5.

9. Nurnberg, HG, Lauriello J, Hensley PL, Parker LM, Keith DS. J Sildenafil for iatrogenic serotonergic antidepressant medicationinduced sexual dysfunction in 4 patients. J Clin Psychiatry 1999;60:33-5.

10. Waldinger MO, Hengeveld MW, Zwinderman AH, Olivier B. Effect of SSRI antidepressants on ejaculation: a double-blind, randomized, placebo-controlled study with fluoxetine, fluvoxamine, paroxetine, and sertraline. J Clin Psychopharmacol 1998;18:274-81.

11. Sachs GS, Guille C. Weight gain associated with the use of psychotropic medications. J Clin Psychiatry 1999;60(supll 21):16-9. 
12. Fava M. Weight gain and antidepressants. J Clin Psychiatry 2000;61(supll 11):37-41.

13. Michelson DM, Amsterdam JD, Quitkin FM, Reimherr FW, Rosenbaum JF, Zajecka J, et al. Changes in weight during a 1-year trial of fluoxetine. Am J Psychiatry 1999;156:1170-6.

14. Thase ME. Antidepressant treatment of the depressed patient with insomnia. J Clin Psychiatry 1999;60(supll 17):28-31.

15. Asnis GM, Chakraburtty A, DuBoff EA, Londborg PD, Rosenberg $\mathrm{R}$, Roth-Schechter B, et al. Zolpidem for persistent insomnia in SSRI-treated depressed patients. J Clin Psychiatry 1999;60:66876.

16. Black K, Shea C, Dursun D, Kutcher S. Selective serotonin reuptake inhibitor discontinuation syndrome: proposed diagnostic criteria. J Psychiatry Neurosc 2000;25:255-61.
17. Beasley CM, Koke SC, Nilsson MR, Gonzales JS. Adverse events and treatment discontinuations in clinical trials of fluoxetine in major depressive disorder: an updated meta-analysis. Clin Ther 2000;22:1319-30.

18. Demyttenaere K, Haddad P. Compliance with antidepressant therapy and antidepressant discontinuation symptoms. Acta Psychiatr Scand 2000;101(supll 403):50-6.

19. Rosenbaum JF, Fava M, Hoog, SL Ascroft RC, Krebs WB. Selective serotonin reuptake inhibitor discontinuation syndrome: a randomized clinical trial. Biol Psychiatry 1998;44:77-87.

20. Schatzberg AF, Haddad P, Kaplan EM, Lejoyeux M, Rosenbaum $\mathrm{JF}$, Young AH, et al. Serotonin reuptake inhibitor discontinuation syndrome: a hypotetical definition. J Clin Psychiatry 1997;58(supll 7):5-10

Correspondência: Aristides Volpato Cordioli

Endereço: Rua Landel de Moura, 1261 - 91920-150 Porto Alegre, RS

Tel.: (0xx11) 3249-54 12-E-mail: arco@ matrix.com.br 